\title{
Mycophenolate mofetil in the treatment of lupus nephritis
}

\author{
Patrick FK Yong ${ }^{1,2}$ \\ David P D'Cruz ${ }^{2}$ \\ 'Department of Clinical Immunology, \\ Kings College Hospital; ${ }^{2}$ The Lupus \\ Research Unit, St Thomas' Hospital, \\ London, UK
}

\begin{abstract}
Lupus nephritis is a complication of systemic lupus erythematosus, which has significant morbidity and mortality. The accepted standard of treatment for severe lupus nephritis is cyclophosphamide for induction of remission. This has significant adverse effects including severe infection and amenorrhea. In addition, although cyclophosphamide induces remission, long-term mortality does not seem to be altered. Mycophenolate mofetil (MMF) is an immunosuppressive agent originally used in solid organ transplantation, which has been compared with cyclophosphamide in trials for lupus nephritis. Randomized trials with MMF have been relatively small, although pooled data seem to suggest that it is at least as effective as cyclophosphamide in inducing remission. In addition, MMF has also been associated with a reduced risk of infection and amenorrhea, although this finding is not universal. MMF appears to be associated with more diarrhea compared with cyclophosphamide. MMF is likely to be a useful treatment for lupus nephritis, although available trial data are limited due to the small size of previous studies. A large trial (the Aspreva Lupus Management Study) is currently underway to attempt to establish the place of MMF in treatment of lupus nephritis.
\end{abstract}

Keywords: mycophenolate mofetil, lupus nephritis, systemic lupus erythematosus

\section{Introduction}

Systemic lupus erythematosus (SLE) is an autoimmune connective tissue disease which can manifest in a variety of ways. Patients with SLE can present with photosensitivity, skin rashes, arthritis, cytopenias, and neurological involvement and frequently possess a plethora of antibodies directed against various components in the cell nucleus, particularly double stranded DNA. In a significant number of patients, SLE can cause nephritis that can result in renal failure with significant morbidity and mortality. Both the prevalence and severity of SLE varies with age, gender, and ethnicity (Danchenko et al 2006). Women of childbearing age are most likely to be affected with SLE. In addition, African and Hispanic ethnicity has been associated with greater prevalence and severity of disease (Bastian et al 2002). This study showed that Caucasians had a $14 \%$ cumulative incidence of nephritis compared with 51\% in African-Americans and $43 \%$ in Hispanics. Another study showed that $10 \%$ of Caucasian patients compared with $27 \%$ of Indo-Asian and $58 \%$ of Afro-Caribbean patients had biopsy proven nephritis (Patel et al 2006).

The assessment of lupus nephritis has improved recently with the updated histological WHO classification by the International Society of Nephrology and the Renal Pathology Society (Weening et al 2004). The updated classification defined class I nephritis as normal glomeruli by light microscopy but with mesangial immune deposits by immunofluorescence; class II as mesangial immune deposits with mesangial hypercellularity; class III as focal glomerulonephritis involving $<50 \%$ of glomeruli with subdivisions for active and sclerotic lesions; class IV nephritis as diffuse glomerulonephritis with $\geq 50 \%$ of total glomeruli affected; class $\mathrm{V}$ as membranous lupus nephritis; and class 
VI as advanced-stage lupus nephritis with $\geq 90 \%$ global glomerulosclerosis. Class IV nephritis was further divided into class IV-S and IV-G with segmental and global involvement as well as subdivisions for active and sclerotic lesions. Classes III, IV, and V are associated with poorer prognoses than Class I and II and usually require active intervention (Yokoyama et al 2004). This updated classification has allowed more meaningful comparison of histological specimens between various centers and standardization of clinical trials by improving reproducibility in classification (Yokoyama et al 2004; Furness and Taub 2006), although at present all published controlled trials were classified using the previous system.

Treatment of severe lupus nephritis usually comprises an induction phase and a maintenance phase. The current accepted standard of care for treatment of proliferative lupus nephritis is monthly high-dose intravenous cyclophosphamide for induction. This regimen was developed in the 1970s and 1980s following trials by the US National Institutes of Health (NIH) (Austin et al 1986; Boumpas et al 1992; Gourley et al 1996). However, a meta-analysis showed that although treatment with cyclophosphamide reduced the risk of doubling of serum creatinine, overall mortality was not altered and there was no significant reduction in the risk of end-stage renal failure (although there was a trend towards better outcomes with cyclophosphamide) (Flanc et al 2004). In addition, these trials included predominantly Caucasian patients despite the higher incidence and severity of lupus nephritis in non-Caucasian populations as well as excluding patients with severely impaired renal function; thus limiting their application to a more generalized lupus nephritis cohort. High-dose cyclophosphamide is also associated with significant adverse effects including an increased infection risk, sterility, secondary malignancy, and hemorrhagic cystitis.

In the 1950s patients with proliferative lupus nephritis rarely lived beyond 5 years (Cameron 1999). This has improved, although cohort studies done between 1992 and 2001 demonstrate that the outcomes in lupus nephritis are still relatively poor. These showed a 5-year mortality of almost $15 \%$ and a 10 -year mortality of almost $25 \%$ (Trager and Ward 2001). The rates of end-stage renal disease were $12 \%$ at 5 years and approximately $25 \%$ at 10 years. These data are significant as they represent "real world" outcomes outside highly specialized centers and suggest that there is still significant ground for improvement of care.

On the background described above, various alternative strategies have been researched in the context of lupus nephritis. Alternative cyclophosphamide regimens have been tried and Houssiau and colleagues compared high dose intravenous cyclophosphamide given monthly for 6 months followed by a further 2 doses 3 months apart (46 patients) against a regimen with six fixed-doses of $500 \mathrm{mg}$ cyclophosphamide every 2 weeks followed by azathioprine (44 patients). At the end of follow-up, there were no significant differences between renal outcomes with a non-significant decrease in infections in patients on the lower dose of cyclophosphamide (Houssiau et al 2002; Houssiau et al 2004). Ten patients in the high-dose group developed severe infections compared with 5 in the low-dose group. Similar numbers of patients in both groups developed hematological and gonadal toxicity, although only 1 patient (in the high-dose group) developed premature menopause. The lack of any other difference in the adverse effects profile was probably due to the small numbers in each group. It should be noted though that the majority of patients included in this study were Caucasian and had preserved renal function at the start of the trial; hence, these results may not be generalized to a non-Caucasian population with significant renal impairment.

In addition to alternative cyclophosphamide regimens, other therapies were also sought and mycophenolate mofetil (MMF) has shown promise in this area. MMF is an immunosuppressant medication that was originally approved by the FDA in 1995 for prevention of renal transplant rejection and has been used in regimens for various other organ transplants. MMF was subsequently used in lupus nephritis initially in uncontrolled cohort studies, which were then followed by randomized controlled trials.

\section{Review of the pharmacology and mode of action of mycophenolate mofetil}

MMF is the 2-morpholinoethyl ester derivative of mycophenolic acid (MPA), a weak organic acid produced by several Penicillium species (Allison and Eugui 2000). MMF has excellent oral bioavailability of $94.1 \%$ in healthy volunteers (Bullingham et al 1998). After absorption, MMF is rapidly converted to its active metabolite, MPA by various plasma, liver and renal esterases. The half life of MPA is 17.9 hours in healthy volunteers and is $97 \%-98 \%$ bound to plasma proteins, primarily albumin, although it is the unbound fraction that is pharmacologically active (Bullingham et al 1998 and Nowak and Shaw 1995). It is mostly excreted through the kidneys as its glucuronide (MPAG). Several factors including renal dysfunction, hypoalbuminemia, accumulation of MPAG and hemoglobin levels have been shown to affect MPA pharmacokinetics and pharmacodynamics (Bullingham et al 1998, Nowak and Shaw 1995, van Hest et al 2006). 
MMF has several effects on the immune system. The best described of these is its selective inhibition of inosine monophosphate dehydrogenase (IMPDH), an enzyme involved in purine biosynthesis. IMPDH exists in two isoforms - type I, which is seen in most cell types and type II, which has greatly increased expression in activated lymphocytes (Natsumeda et al 1990). MMF inhibits the type II isoform nearly 5 times as much compared with the type I isoform, hence conferring its specificity for activated lymphocytes (Carr et al 1993).

In addition to this, further specificity is conferred by MMF's mode of action. Two mechanisms exist for generation of purines (which are required to make up DNA and RNA) the salvage pathway and the de novo pathway. Most cell types can employ either pathway but lymphocytes are unique in their complete dependence on the de novo pathway for purine synthesis. IMDPH is only absolutely required in the de novo pathway (Allison and Eugui 2000), thus resulting in a net decrease in DNA synthesis and inhibition of cellular proliferation predominantly in activated lymphocytes.

Apart from its effects on proliferation of activated lymphocytes, various other properties have been ascribed to MMF. In vitro data have shown that MMF inhibits the proliferation of fibroblasts and vascular smooth muscle cells (Morath et al 2006) as well as inhibiting collagen deposition, tubular cell proliferation, and interstitial fibrosis (Roos et al 2007). In addition, MMF interferes with antibody production by B cells (Allison et al 1991) and adhesion molecule expression (Blaheta et al 1999), thus limiting the immune cell-cell interactions required for full activation. Dendritic cells, which are the principal cell responsible for activating naïve T cells, are also affected by MMF (Colic et al 2003), raising the hypothesis that MMF may contribute to inducing a state of immune tolerance towards previously reactive antigens (Lagaraine and Lebranchu 2003). MMF has also been thought to play a role in reducing inflammation via suppression of inducible nitric oxide synthase (Senda et al 1995).

\section{Animal models}

MMF has been shown to improve outcomes in both the MRL/lpr and NZB $\times$ NZW F1 murine models of lupus nephritis. Eight-week-old MRL/lpr mice treated with 90 $\mathrm{mg} / \mathrm{kg}$ MMF daily had a reduced likelihood of developing albuminuria at 23 weeks ( 22 vs $88 \%$ in controls), histologically less severe glomerulonephritis, and less immunoglobulin and C3 deposits in glomerular capillary walls compared with control mice (van Bruggen et al 1998). Three-monthold MRL/lpr mice with established glomerulonephritis treated with $100 \mathrm{mg} / \mathrm{kg}$ MMF daily had prolonged survival, reduced incidence of hematuria and albuminuria, and reduced numbers of B cells and IgG anti-dsDNA antibodies compared with control mice (Jonsson et al 1999). The same study also demonstrated that cyclophosphamide had similar efficacy to MMF treatment in that setting.

Improvements in disease outcomes have also been seen in the NZB $\times$ NZW F1 model. Three-month-old mice treated with MMF (60 mg/kg daily) until death were less likely to develop proteinuria, had improved renal function, and better survival than control mice ( $80 \%$ vs $50 \%$ alive at 9.5 months) (Corna et al 1997). In another study, MMF at a higher dose of $200 \mathrm{mg} / \mathrm{kg}$ daily resulted in 100\% survival compared with $10 \%$ in controls, and also suppressed the development of albuminuria and anti-dsDNA antibodies (McMurray et al 1998). Mice treated with MMF (100 or $30 \mathrm{mg} / \mathrm{kg}$ daily) from the age of 3 months (prior to renal immune complex deposition but at the time when serum autoantibodies are present) had improved survival compared with control $(>90 \%$ in both groups of MMF-treated mice vs $42 \%$ in controls at 42 weeks) (Ramos et al 2003). Of note, direct inhibition of autoantibody production was only seen at the higher dose of MMF, whereas qualitative changes with a reduction in total and antigen-specific IgG2a were seen at the lower dose of MMF, suggesting that this might be one of the mechanisms resulting in disease amelioration.

MMF in combination with a cyclooxygenase-2 (COX-2) inhibitor has also been used in this model starting at the age of five months, at the time of renal immune complex deposition (Zoja et al 2001). MMF alone (60 mg/kg daily) improved animal survival (93\% vs $53 \%$ at 8 months, $67 \%$ vs $47 \%$ at 9 months) and limited renal damage compared with control. These benefits were significantly increased in the group of animals treated with a combination of MMF and the COX-2 inhibitor, which was used due to the previous finding of increased COX-2 derived thromboxane-A2 in lupus nephritis, thought to contribute to renal injury.

In addition to the role of thromboxane-A 2 in lupus nephritis, two groups of investigators have investigated the effects of MMF on inducible nitric oxide synthase (iNOS), which has been implicated in pathogenesis of lupus nephritis through generation of excess nitric oxide. Benefits were seen in survival (Lui et al 2002), levels of proteinuria and histological severity in MMF-treated MRL/lpr mice (Yu et al 2001; Lui et al 2002). Renal cortical levels of iNOS mRNA and urinary nitrate production were reduced in one study (Yu et al 2001) whereas the other study did not find any difference in intrarenal nitric oxide production, iNOS protein and mRNA levels and urinary nitrite/nitrate production at 
12 weeks although there was a transient decrease in urinary nitrite/nitrate production at 8 weeks (Lui et al 2002).

In summary, MMF has been shown to be beneficial in murine models of lupus although its exact mechanism of action remains to be fully determined.

\section{Clinical trials of mycophenolate mofetil in lupus nephritis}

To date, there have been 8 published reports of 6 randomized controlled trials ( 2 in abstract form only) of the use of MMF in lupus nephritis (Chan et al 2000; Ye et al 2001; Contreras et al 2004; Flores-Suarez and Villa 2004; Chan et al 2005; Contreras et al 2005; Ginzler et al 2005; Ong et al 2005). There have also been a significant number of cohort studies describing the use of MMF in SLE, although not all patients in these studies had lupus nephritis. There have also been 3 meta-analyses and systematic reviews analyzing the available data. It should be noted that when assessing these controlled studies, there are limitations in the quality of the data available. All of these studies were open label and had unblinded assessors. In addition, most trials did not use allocation concealment or an intention-to-treat analysis. This is reflected in the Jadad (quality) scores for the trials of 2 or 3 (Walsh et al 2007).

There were also differences in the histology in the various trials. All trials included patients with proliferative lupus nephritis (Class III and IV, although some only had patients with Class IV disease). Some of the studies included patients with membranous nephritis (with and without proliferative change) which has a different clinical course to proliferative nephritis and it is not clear how inclusion of these patients could affect trial results. There are also limited available data on the use of MMF purely restricted to Class V nephritis; this is discussed further below.

Chan et al randomized 21 Chinese patients with diffuse proliferative lupus nephritis (WHO Class IV) each to either oral cyclophosphamide $(2.5 \mathrm{mg} / \mathrm{kg} /$ day $)$ replaced by azathioprine at 6 months or oral MMF $2 \mathrm{~g}$ daily for 6 months, followed by MMF $1 \mathrm{~g}$ daily for a further 6 months and then replaced by azathioprine (Chan et al 2000). All patients were also treated concurrently with prednisolone. Remission was defined by urinary protein excretion and creatinine clearance. $81 \%$ of patients treated with MMF had complete remission and $14 \%$ had a partial remission compared with $76 \%$ and $14 \%$ of patients treated with cyclophosphamide. Relapse rates were $15 \%$ and $11 \%$ respectively. The authors concluded that MMF and prednisolone were at least as effective as oral cyclophosphamide and prednisolone.
In a subsequent extended follow-up study, with a median follow-up of 63 months, they investigated the role of MMF in induction-maintenance of lupus nephritis (Chan et al 2005). The original protocol using MMF was modified to treatment with MMF $2 \mathrm{~g}$ daily for 6 months, followed by $1.5 \mathrm{~g}$ daily for 6 months and then $1 \mathrm{~g}$ daily. Further patients were also recruited, increasing total numbers to 32 patients in the MMF group and 30 patients in the cyclophosphamide-azathioprine group. Greater than $90 \%$ of patients in both groups had either a complete or partial response to induction treatment. Over the follow-up period, there was no significant difference between the risk of relapse and relapse-free survival between those treated with cyclophosphamide and MMF and between those treated with MMF for 12 or greater than 24 months. This provided evidence that MMF and prednisolone was an effective treatment for induction-maintenance in lupus nephritis.

Criticisms levelled at the study have included the use of oral cyclophosphamide compared with MMF, when the standard of care is intravenous cyclophosphamide and the relatively high cumulative dose of cyclophosphamide. In addition, patients included in the study also only had mild renal impairment at worst and were drawn solely from a Chinese population.

Contreras et al investigated the role of MMF as a sequential therapy following induction with up to a maximum of seven monthly intravenous boluses of cyclophosphamide $\left(0.5-1.0 \mathrm{~g} / \mathrm{m}^{2}\right.$ body surface area) (Contreras et al 2004; Contreras et al 2005). Fifty-nine patients with lupus nephritis (12 with WHO class III, 46 with class IV and 1 with class $\mathrm{Vb})$ were randomized to 3-monthly intravenous cyclophosphamide, oral azathioprine (1-3 mg/kg daily), or oral MMF (500-3000 mg daily) following induction. In addition, all patients received corticosteroids. The study population comprised 30 Hispanic, 27 black, and 3 white patients. The event-free survival rate for a composite endpoint of death or chronic renal failure was significantly higher in the MMF and azathioprine groups compared with the cyclophosphamide group and the rate of relapse-free survival was higher in the MMF group compared with the cyclophosphamide group. Five patients died (4 in the cyclophosphamide group, 1 in the MMF group) and 5 patients developed chronic renal failure ( 3 in the cyclophosphamide group and 1 each in the remaining 2 groups) during maintenance therapy. The authors concluded that short term therapy with intravenous cyclophosphamide followed by maintenance therapy with MMF or azathioprine was more effective than long-term therapy with intravenous cyclophosphamide. 
However, the number of patients with longer-term data was limited with 27 patients followed to 36 months and only 5 were followed to 72 months; thus providing limited data on the longer-term outcomes of MMF use in maintenance therapy.

In the largest published study of MMF in lupus nephritis to date, Ginzler et al subsequently reported results of a 24 week non-inferiority trial comparing oral MMF (1 g daily, increased to $3 \mathrm{~g}$ daily) against monthly intravenous cyclophosphamide $\left(0.5 \mathrm{~g} / \mathrm{m}^{2}\right.$ body surface area, increased to $1 \mathrm{~g} / \mathrm{m}^{2}$ ) (Ginzler et al 2005). Seventy-one patients were randomized to MMF and 69 patients to cyclophosphamide and the primary endpoint of the study was complete remission (defined as normalization of abnormal renal measurements and maintenance of baseline renal measurements) at 24 weeks. A secondary endpoint was partial remission at 24 weeks, defined as improvement of $50 \%$ of all abnormal renal measurements, without worsening of any measurement. Seventy-nine patients were black with 28 Hispanic and 24 white patients. Twenty-two had WHO class III nephritis, 76 had class IV, and 27 had class V. In their study, $22.5 \%$ of patients receiving MMF compared with $5.8 \%$ of patients receiving cyclophosphamide had a complete remission. There was no significant difference in patients who achieved a partial remission (29.6\% in the MMF group compared with $24.6 \%$ in the cyclophosphamide group). The authors concluded that this demonstrated that MMF was superior at inducing remission compared with intravenous cyclophosphamide.

The patients in this study also had relatively well preserved renal function at the start despite more than $50 \%$ of patients having class IV nephritis. The question has also been raised as to why the study protocol used a slowly increasing dose of MMF as opposed to more aggressive therapy with gradual tapering. Activity and chronicity indices were not reported.

The remaining published randomized trial was undertaken by Ong et al where they randomized patients to induction therapy with intravenous cyclophosphamide $0.75-1 \mathrm{~g} / \mathrm{m}^{2}$ body surface area (25 patients) or oral MMF $2 \mathrm{~g}$ daily (19 patients) for 6 months, both with corticosteroids (Ong et al 2005). The primary endpoint was remission of lupus nephritis (combined partial and complete remission) at 6 months. Patients had either WHO class III or IV nephritis with or without membranous change; and were made up of 22 Malay, 20 Chinese, and 2 patients of other ethnicity. Similar numbers of patients in each group achieved remission (52\% in the cyclophosphamide group and $58 \%$ in the MMF group) and complete remission
(12\% and $26 \%$ respectively). There was no statistical difference between the various groups. Attending physicians were free to prescribe their choice of immunosuppressive therapy after the initial 6-month period and this comprised azathioprine, ciclosporin, and further intravenous cyclophosphamide. Further long-term follow-up data (up to 48 months) showed no significant differences in patient kidney survival between the two randomized groups. The authors concluded that MMF with corticosteroids was as effective as cyclophosphamide in induction therapy.

Similar to the Chan et al study, this study also did not include any patients of African, Caucasian, or Hispanic origin. It should be noted that in both the Ginzler et al and Ong et al studies, the primary outcome measure was remission (including improvement of proteinuria) at 6 months. In view of this, caution should be employed when interpreting the results as proteinuria can take a prolonged period of time to resolve in severe nephritis; and late-stage renal failure can occur beyond the 6-month period.

There are also two further randomized trials published in abstract form only. Ye et al randomized 90 patients with severe SLE (with one or more of lung, renal, central nervous system, hemolytic anemia or vasculitic complications) to either monthly intravenous cyclophosphamide for 6-12 months or MMF $1.5 \mathrm{~g}$ daily tapered to $1 \mathrm{~g}$ daily at 3 months and $0.5-0.75 \mathrm{~g}$ daily after a further 3 months (Ye et al 2001). The WHO class was not stated in the abstract. MMF was found to significantly improve clinical and laboratory parameters, although no direct comparison with cyclophosphamide was made in the abstract. There was, however, a significant difference in the incidence of adverse effects (gastrointestinal tract reactions, infection, leukopenia, hair loss, liver dysfunction, and menopause) in the MMF group. FloresSuarez randomized 10 patients with class IV or V nephritis to either MMF up to $2 \mathrm{~g}$ daily or monthly intravenous cyclophosphamide (Flores-Suarez and Villa 2004). MMF was as effective as intravenous cyclophosphamide and significantly more patients on MMF achieved partial remission. Infections appeared to be milder in the MMF group.

In addition to the randomized trials, there have been several uncontrolled open-label cohort studies describing the use of MMF for a variety of disease manifestations in SLE. These studies have been relatively small, with the majority having numbers generally ranging between 10 and 30 patients with 1 single study describing a cohort of 86 patients. The cohort studies are listed in Moore and Derry 2006. Although most of these studies, showed improvement in the patients treated with MMF, it is difficult to draw definitive conclusions as no comparison 
with standard therapy was made. However, Moore and Derry did pool together data from these various studies in their metaanalysis (discussed further below).

Due to the relatively small number of patients in studies to date and some of the uncertainties surrounding the use of MMF, the Aspreva Lupus Management Study (ALMS) was undertaken to contribute further data in both induction and maintenance therapies for lupus nephritis (Sinclair et al 2007). The study recruited 370 multi-racial patients from approximately 100 centers worldwide. Patients enrolled were between 12 and 75 years of age with SLE diagnosed according to the revised American College of Rheumatology criteria; and had a histological diagnosis of Class III, Class IV-S, or IV-G, or Class V lupus nephritis or a mixture of these within 6 months prior to randomization. In addition, participants had to have active nephritis defined as: proteinuria $\geq 1 \mathrm{~g} / 24$ hours or elevated serum creatinine $(>1.3 \mathrm{mg} / \mathrm{dL})$ or active urinary sediment in patients with Class IV-S or IV-G disease; and proteinuria $\geq 2 \mathrm{~g} / 24$ hours or elevated serum creatinine $(>1.3 \mathrm{mg} / \mathrm{dL})$ in patients with Class III or V disease. Patients were excluded if they were receiving continuous dialysis for more than 2 weeks prior to randomization or had received (or was due to receive) a renal transplant. Patients who also had induction or maintenance immunosuppressive therapy within 12 months or 2 weeks respectively were also excluded. The inclusion criteria were broader than previous trials and allowed for more severely ill patients to be recruited as well as investigating treatment of class V nephritis. It was also hoped that the ALMS study would provide additional information on the relevance of the histological classification of lupus nephritis.

In the trial, patients were randomized to either oral MMF (target dose of $1.5 \mathrm{~g}$ bd) or intravenous cyclophosphamide (every 4 weeks for 6 infusions; starting at $0.75 \mathrm{mg} / \mathrm{m}^{2}$ for the first month with subsequent doses at $0.5-1.0 \mathrm{mg} / \mathrm{m}^{2}$ ) for 24 weeks of induction therapy; followed by re-randomization to either oral MMF (1 g bd) or oral azathioprine $(2 \mathrm{mg} / \mathrm{kg}$ daily) for maintenance if they achieved treatment response. All patients also received a concomitant dose of oral steroids pre-defined by the trial protocol.

Results of the induction phase have now been reported in abstract form (Ginzler et al 2007). The study did not meet its primary objective of showing a superior response rate with MMF compared with intravenous cyclophosphamide. Overall, 56.2\% (104/185) of patients on MMF and 53.0\% $(98 / 185)$ of patients on cyclosphosphamide had a response to treatment (defined as decrease in proteinuria and improvement/stabilization in serum creatinine). These rates were not significantly different. However, on further analysis by racial group, significant differences in response rates were found: 147/370 patients reported themselves as Caucasian, 123 patients were Asian, and 100 patients belonged to "other" - a group comprising mostly black and mixed-race patients. Across all racial groups, 131 patients described their ethnicity as Hispanic. Response rates for MMF and cyclophosphamide were similar for Caucasian and Asian patients; however, there were significant differences for Hispanic patients (60.9\% response with MMF vs 38.8\% with cyclosphophamide) and those classified as "other" (60.4\% response with MMF vs 38.5\% with cyclophosphamide). No differences at baseline between the various groups were found to explain this variation.

The rate of adverse events was similar between the two groups during the 24-week induction phase. There were 24 withdrawals from the MMF group (12 due to infection) and 13 from the cyclophosphamide group (4 due to infection). There were 9 deaths in the MMF group ( 7 due to infection) and 5 in the cyclophosphamide group (2 due to infection and 2 due to SLE).

\section{Class V (membranous) lupus nephritis}

Membranous lupus nephritis occurs in about $20 \%$ of patients with lupus nephritis and represents a distinct entity histopathologically. Clinically, when compared with patients with proliferative lupus nephritis, patients with membranous lupus nephritis tend to frequently have nephrotic range proteinuria, with more variable and more slowly progressive loss of renal function. There can, however, be considerable overlap in the clinical features and membranous lupus nephritis can occur with proliferative nephritis (Austin and Illei 2005). At present, there is limited evidence available on the best approach to treat membranous lupus nephritis. The relatively indolent course in membranous lupus nephritis had been the main argument against using potent immunosuppressants, although more recently, it has been noted that there are significant risks associated with prolonged nephrotic syndrome. Consequently, some have now contended that aggressive therapy should be considered to reduce nephrotic proteinuria to prevent the long-term complications of cardiovascular disease as a result of dyslipidemia and pro-thrombotic tendency. Nonetheless, although there is greater acknowledgment of these risks, there remain few data on the long-term benefits and risks of treating membranous lupus nephritis with potent immunosuppressants (Austin and Illei 2005).

Several small uncontrolled observational studies of MMF use in membranous lupus nephritis have been published. 
Spetie et al reported their experience of MMF in 13 consecutive treatment naïve patients with membranous lupus nephritis (12 had pure class V nephritis and 1 had class III and V), who had a mean 24-hour urine protein:creatinine ratio of 5.1 (Spetie et al 2004). These patients were treated with MMF (mean dose $1173 \mathrm{mg}$ daily) and prednisolone (mean dose $31 \mathrm{mg}$ daily), in addition to other measures to reduce proteinuria (including an ACE inhibitor and/or anigotensin receptor blocker). Ten of their patients had achieved complete or partial remission at 6 months and at the end of their study period (mean follow-up of 16 months), 9 patients were in complete remission and 11 had protein:creatinine ratios of $<0.8$. During the 208 patient months of follow-up, the only serious complication was of histoplasma pneumonia in one patient.

In their retrospective study, Kapitsinou et al reported on their cohort of patients, 6 of whom had membranous nephropathy ( 3 with pure membranous nephropathy, 1 with additional focal proliferative change, and 2 with additional diffuse proliferative change) (Kapitsinou et al 2004). Patients were treated with MMF $1 \mathrm{~g}$ twice daily and steroids for a mean duration of 14.8 months; the mean duration of nephritis prior to MMF treatment was 75 months. Mean proteinuria decreased from $1.9 \mathrm{~g}$ to $1 \mathrm{~g}$ daily, although this was not significant, and there was no significant difference in mean creatinine clearance. Four of the group had treatment failure defined as no remission at the end of follow-up.

Karim et al subsequently reported on 10 patients with predominantly membranous lupus nephritis (6 with additional focal proliferative change and 4 with pure membranous change) showing a significant improvement in urinary protein excretion and serum albumin (Karim et al 2005). Nine of the patients had received previous immunosuppression and all of them were on antihypertensive agents prior to starting MMF. Patients were treated with MMF (doses between 1 and $2.5 \mathrm{~g}$ daily) for a mean time of 18.8 months. 24-hour urine protein excretion reduced from a median $2.26 \mathrm{~g}$ to $0.66 \mathrm{~g}$ with a concomitant rise in serum albumin from a median of $29.5 \mathrm{~g} / \mathrm{L}$ to $33.5 \mathrm{~g} / \mathrm{L}$ at the end of follow-up. Serum creatinine was not significantly affected. During the study period, 2 patients had infectious complications, 5 had gastrointestinal symptoms, and 1 patient needed to discontinue MMF.

More recently, Kasitanon et al reported a retrospective series of 29 cases of membranous nephropathy treated with MMF, analyzing the differences between those with and without concurrent proliferative disease; something not reported in previous papers (Kasitanon et al 2008). Ten patients had pure membranous nephropathy and the remaining 19 had mixed membranous and proliferative nephritis; all patients had not received any immunosuppression apart from oral steroids at the start of the study. Patients were treated with $2 \mathrm{~g}$ daily of MMF, increased to $3 \mathrm{~g}$ daily after a month if tolerated. Patients in the group with pure membranous nephropathy were followed for a mean of 40.4 months compared with 23.6 months in those with a mixed nephropathy. The authors found no significant difference in renal outcomes between the two groups. At 12 months, 4/10 patients with pure membranous nephropathy and 7/19 patients with mixed nephropathy had achieved complete remission whereas $1 / 10$ in the first group and $2 / 19$ in the second had a worsening of renal disease; the remainder of the patients had no change in disease status. Overall, about $40 \%$ of their patients, particularly those with mild proteinuria responded to MMF treatment. Three patients developed infective complications (lobar pneumonia and septic arthritis) with no cases of herpes zoster.

From the limited number of studies, it would seem that MMF has a degree of efficacy in membranous lupus nephritis, with some studies reporting greater success than others although the patient cohorts in them are not directly comparable. However, there are no head-to-head comparisons with other agents; the study by Ginzler et al did include 27 patients with class $\mathrm{V}$ nephritis but no further sub-analysis of this group was published (Ginzler et al 2005). Until more trials are done in membranous nephritis, it is difficult to comment at present on how MMF compares with other immunosuppressive therapies in this area.

\section{Use of MMF in pediatric lupus nephritis}

At present, there are no published controlled trials of the use of MMF in pediatric SLE. Fu and Liu described the use of MMF in two Chinese children with lupus nephritis who were refractory to previous cyclophosphamide and ciclosporin therapy (Fu and Liu 2001). All clinical symptoms and serum autoantibodies had become negative after 11-12 months of treatment. A case series of 11 children was also published in the same year (Buratti et al 2001). In that paper, patients had lupus nephritis refractory to treatment with high dose oral or intravenous prednisolone, cyclophosphamide and/or azathioprine and were treated with a mean dose of $22 \mathrm{mg} / \mathrm{kg} /$ day for a mean duration of 9.8 months. In 4 patients with class $\mathrm{V}$ membranous lupus nephritis, renal function normalized although little effect was seen in the children with class IV proliferative glomerulonephritis. In addition, adverse events were seen in 8 out of $11(73 \%)$ patients, including infections, leucopenia, nausea, pruritus, headache, and fatigue. 
Pecoraro et al published an abstract of 14 children with lupus nephritis (mean age 12.4 years) with more than $3 \mathrm{~g}$ of proteinuria daily and normal renal function who were treated with IV methyprednisolone followed by MMF (mean dose $29 \mathrm{mg} / \mathrm{kg}$ daily) and oral prednisolone for 2 years (Pecoraro et al 2005). Seven children had class IV nephritis and the remainder had a mixture of other types. Repeat renal biopsies were done after 2 years and the authors found a reduction in inflammatory cells in all biopsies as well as resolution of proteinuria in all cases, without any significant hematologic or gastrointestinal events.

In a retrospective study, Lau et al reported on 44 predominantly African American children with a mean age at biopsy of 14.2 years (Lau et al 2006). Only five patients in their cohort received MMF (1 with class III nephritis and 2 each with class IV and V nephritis) and no further analysis by treatment was undertaken due to the small numbers of patients. However, the authors did note that those with class $\mathrm{V}$ nephritis did have a lower mean urine protein: creatinine ratio at 12 months than those treated with corticosteroids alone.

In a review, Adams et al however, noted that in their practise, MMF was less effective and was associated with more adverse events compared with quarterly intravenous cyclosphosphamide (Adams et al 2006). In addition, they had had no patients who had managed to maintain a cyclophosphamide-induced remission with MMF, although numerical data were not provided. This was thought to be due to poor compliance secondary to gastrointestinal side effects. They recommended that cyclophosphamide should continue to be used in children with class III or IV lupus nephritis. However, Paredes suggested that MMF could be considered for mild to moderate nephritis with preserved renal function where fertility was an issue and oral compliance could be ascertained; while maintaining the use of IV cyclophosphamide for the most unwell children (Paredes 2007). In view of the paucity of data on the use of MMF in children, it would seem reasonable to take this approach at present until further trials are conducted.

\section{Safety and tolerability}

In general, MMF has been reported to be relatively well tolerated. The principal adverse effects include gastrointestinal symptoms particularly diarrhea, nausea and vomiting and abdominal cramps. There is a suggestion that the gastrointestinal side effects may occur more frequently in the transplant setting compared with its use in inflammatory disease (Goldblum 1993). Side effects typically occur early in the course of treatment and tend to decrease with continued use. Some of the strategies to reduce the incidence of gastrointestinal side effects include divided doses or administration of the drug with food. In addition, hematologic (primarily leucopenia) and infection-related adverse events have also been reported, although it is unclear if the data obtained from its use in the transplant setting can be directly extrapolated due to the higher immunosuppressive burden in transplant patients. Hence, to determine the side effect profile in SLE it is necessary to evaluate the safety of MMF in trials specific for this condition.

In their trial, Chan et al reported that significantly fewer patients treated with MMF developed infections that required antibiotic treatment $(12.5 \%$ vs $40 \%$ for the cyclophosphamide-azathioprine group) or hospitalization ( $6.3 \%$ vs $30 \%$ for the cyclophosphamide-azathioprine group) (Chan et al 2005). The incidence of infection was 1 in 234.0 patient-months in the MMF group compared with 1 in 102.5 months in the cyclophosphamide-azathioprine group. Four patients in the cyclophosphamide-azathioprine group died or developed end stage renal failure compared with none in the MMF group, although this difference was not statistically significant. Other adverse events that were significantly lower in the MMF group were leucopenia $\left(<4 \times 10^{9} / \mathrm{L}\right)$, severe hair loss, and amenorrhea. There was no significant difference between the two groups for gastrointestinal upset, withdrawal due to side effects, or infection with herpes zoster.

In the study by Contreras et al the authors concluded that MMF and azathioprine were safer than intravenous cyclophosphamide for long-term maintenance (Contreras et al 2004, 2005). Hospitalization rates were significantly lower in the MMF and azathioprine groups (1 hospital-day per patient-year) compared with the cyclophosphamide group (10 hospital-days per patient-year). The rate of severe infections and total infections was lower in both the MMF and azathioprine groups compared with the cyclophosphamide group. There was also significantly less amenorrhea ( $6 \%$ and $8 \%$ vs $32 \%$ ), nausea and vomiting when the MMF and azathioprine groups were compared with the cyclophosphamide group. There were no significant differences in the incidence of diarrhea or leucopenia between the various groups.

Ginzler et al also reported fewer hospitalizations and severe infections in their MMF group. 6/75 patients in the cyclophosphamide group developed severe infection (necrotizing fasciitis, gram-negative sepsis, pneumonia and lung abscess) compared with $1 / 83$ in the MMF group (relative risk $[R R]$ of $0.36, p=0.03$ ) (Ginzler et al 2005). Amenorrhea occurred in 2 patients receiving cyclophosphamide and none on MMF. Hematologic side effects were reported to be 
uncommon with 5 patients in the MMF group and 14 patients in the cyclophosphamide group developing sustained lymphopenia. Diarrhea was more common in the MMF group, with 15 patients developing this compared with only 2 in the cyclophosphamide group.

Two deaths occurred in the cyclophosphamide group with none occurring in the MMF group. One of these deaths was due to a cerebral hemorrhage occurring shortly after the first dose of cyclophosphamide and the second death occurred after 2 doses of cyclophosphamide and was reported to be due to active SLE and sepsis. A third patient who was assigned to cyclophosphamide treatment declined therapy and subsequently died.

The study by Ong et al unlike the previous three trials reported no significant difference in their rate of adverse events at 6 months (Ong et al 2005). There was no difference between the rates of infections, with 3 patients in each group developing pneumonia or septicemia and another 3 patients developing herpes zoster. There was also no difference in the rates of diarrhea. Of patients in the cyclophosphamide group, $52 \%$ developed leucopenia $\left(<3.5 \times 10^{9} / \mathrm{L}\right)$ compared with $36.8 \%$ of patients on MMF although this was not statistically significant. A single patient in the cyclophosphamide group developed amenorrhea and another patient discontinued therapy due to persistent leucopenia. At the end of the follow-up period of 36 months, there had been 1 death in each group.

It should be noted that in the transplant setting, however there have been reports of increased infection rates with herpesviruses, associated with MMF use. Most of the data available on this are on cytomegalovirus (CMV) infection; and the results have been conflicting, although overall MMF does seem to be associated with a modest increase (Wang et al 2004; Song et al 2006). It is unclear whether this increase is due specifically to MMF use or whether it represents overall immunosuppressive burden. A similar increase has not been noted in the studies of lupus nephritis, where overall immunosuppressive burden is lower. There are limited controlled data on the other herpes viruses, although there is a suggestion that herpes zoster occurs more frequently in liver transplant recipients, but not other organ transplants (Gourishankar et al 2004).

\section{Enteric-coated mycophenolate sodium}

In view of the frequent gastrointestinal side effects with $\mathrm{MMF}$, an alternative enteric coated mycophenolate sodium preparation has been introduced. In the renal transplant setting, trials have shown equivalent safety and efficacy to conventional MMF (Salvadori et al 2004; Budde et al 2006) with a reduced incidence in gastrointestinal-symptom burden (Chan et al 2006). There is only a single observational study on the use of mycophenolate sodium in lupus nephritis, with 16 patients receiving this as induction therapy (Kitiyakara et al 2008). Eight of them achieved complete remission and 4 had a partial remission. Gastrointestinal side effects were reported to be low, although there was no direct control group. However, despite the lack of specific data in lupus nephritis, there is no reason to expect that this would be significantly different from the findings in the transplant setting.

\section{Pregnancy and lactation}

One of the factors limiting the use of MMF is its teratogenicity. MMF has been shown to cause fetal malformations in rats and rabbits, resulting in cardiovascular, renal, and central nervous system defects, in the absence of maternal toxicity at doses equivalent to half or less of the recommended treatment dose (EMEA 2008). Fetal malformations in humans have also been reported, although no controlled trial data are available. There had been no clear pattern of malformations noted, although a recent report suggesting a possible characteristic phenotype with cleft lip and palate, microtia with atresia of the external auditory canal, micrognathia, and hypertelorism has been published (Perez-Aytes et al 2008). In view of its teratogenicity, the product data sheet states mycophenolate is currently not recommended for use in pregnancy and effective contraception needs to be maintained while on treatment and for up to 6 weeks after discontinuation (EMEA 2008).

MMF has also been shown to be expressed in the breast milk of lactating rats, although there are no data in humans. Consequently, the manufacturer has advised avoidance of MMF in breastfeeding mothers (EMEA 2008).

\section{Meta-analyses and systematic reviews}

In general, studies conducted on lupus nephritis have been few and relatively small. The total number of patients recruited in the 6 randomized studies described above was 370. In addition, there are only about 200-300 patients in randomized studies involving cyclophosphamide and azathioprine (Flanc et al 2004), the most widely used therapies at present. Consequently, meta-analyses are limited as the studies available for inclusion are small, short-term, and of limited methodological quality. Nonetheless based on available data, 3 meta-analyses on the use of MMF in lupus nephritis have been published to date (Moore and Derry 2006; Walsh et al 2007; Zhu et al 2007). 
Moore and Derry used 5 of the randomized studies (Chan et al 2000, 2005; Contreras et al 2004, 2005; Flores-Suarez and Villa 2004; Ginzler et al 2005; Ong et al 2005) totalling 306 patients, deemed to have sufficient data for analysis (Moore and Derry 2006). They pooled data from all these studies to calculate the efficacy rates (defined by complete response, complete or partial response and subsequent relapse) and adverse event rates (death, hospital admission, adverse events discontinuation, all infections, serious infections, leucopenia, amenorrhea, hair loss, and diarrhea). No distinction was made between studies investigating the use of MMF in induction, maintenance or both. They found that MMF was significantly more likely to result in a complete response (36\% of patients vs $23 \%$ with cyclophosphamide, relative benefit 1.5$)$ and complete or partial response $(66 \%$ of patients vs $54 \%$ with cyclophosphamide, relative benefit 1.2) than cyclophosphamide. There was no significant difference between the two therapies for risk of subsequent relapse. They calculated that the number needed to treat with MMF was 7.6 (95\% confidence interval [CI] 4.2-43) for one additional complete response and 8.0 for 1 additional complete or partial response.

The combined data for all randomized trials also showed a significantly lower incidence of death $(0.7 \%$ with MMF vs $7.8 \%$ with cyclophosphamide; with 1 less death occurring for every 14 patients treated with MMF). Other adverse events that occurred significantly less frequently with MMF were all infections, serious infections, leucopenia, amenorrhea and hair loss. Diarrhea occurred significantly more frequently with MMF (16\% vs 4\% with cyclophosphamide, RR 4.0). There were no significant differences between the rates of discontinuation due to adverse events.

In addition to the randomized trials, the authors also identified 11 fully published cohort studies and a further 7 as abstracts. The amount of data reported in these studies was variable and of these, only 10 studies treated patients solely for lupus nephritis. In addition, only 5 of these documented histology on biopsy, whereas the rest used deteriorating renal function, rising dsDNA titers, or inadequate control on standard immunosuppression. Complete or partial response to therapy was reported in only 7 of those studies, giving a total of 151 patients. Of those patients, 121 (80\%) had a complete or partial response following treatment with MMF. Overall, there was a $14 \%$ rate for discontinuation due to adverse events and a $10 \%$ rate of discontinuation due to lack of efficacy. All infections were common, affecting $23 \%$ of patients but serious infection occurred in only about $4 \%$. Gastrointestinal events affected about $30 \%$ of patients and there was no occurrence of amenorrhea. There was only a single death (after serious infection) reported in all the cohort studies.

Zhu et al, in another meta-analysis, utilized only data from the 3 published trials (and not the abstract by FloresSuarez et al 2004) comparing MMF with cyclophosphamide for induction treatment of lupus nephritis (Zhu et al 2007). They found no significant difference between the rates of MMF or cyclophosphamide to induce complete remission, partial remission and overall remission. MMF was significantly less likely to cause infection (RR 0.65). There was a non-significant decrease in amenorrhea (RR $0.22,95 \% \mathrm{CI}$ $0.04-1.22$ ) and leucopenia (RR $0.61,95 \%$ CI $0.37-1.03$ ) with MMF. MMF was also more likely to cause gastrointestinal symptoms, but again this did not reach statistical significance (RR 1.33, 95\% CI 0.97-1.84). There was also no significant difference between the risk of developing end-stage renal failure or death between those treated with MMF and cyclophosphamide at induction.

They further performed a sensitivity analysis by excluding the Chan trial as it had used oral cyclophosphamide for induction. This was on the basis that oral cyclophosphamide differed from intravenous cyclophosphamide in some of its effects. On exclusion of this trial, Zhu et al then found that the rate of complete remission was significantly higher in the MMF group (relative benefit 3.10, 95\% CI 1.38-7.01) and that the incidence of leucopenia was significantly lower. They did not find any difference in the remaining results.

In addition, Zhu et al also analyzed the trials that compared MMF with azathioprine for maintenance treatment of lupus nephritis. They found no significant difference between MMF and azathioprine with regard to the incidence of death, end stage renal failure, disease relapse and doubling of serum creatinine. There was a higher incidence of gastrointestinal symptoms in the MMF group, but a lower incidence of leucopenia.

In the most recent meta-analysis, Walsh et al again compared only the studies that randomized patients for induction therapy (Walsh et al 2007). They utilized the studies analyzed by Zhu et al as well as the abstract published by Flores-Suarez et al 2004, giving a total of 268 patients. They noted that 3 out of the 4 studies failed to show any significant difference in induction of remission but that the pooled data showed a RR of 0.70 (95\% CI 0.54-0.90) for failure of MMF to induce remission compared with cyclophosphamide. They also analyzed a composite outcome of death or end-stage renal failure. This showed a non-significant difference at the end of the prespecified study duration, although the RR was lower with MMF compared with cyclophosphamide 
(RR 0.35, 95\% CI 0.10-1.22). However, on using extended follow-up data, this lower risk with MMF was significant (RR 0.44, 95\% CI 0.23-0.87).

Walsh et al also concluded that in general there were too few adverse events for most categories to perform analysis, apart from infection, which had a non-significantly lower risk for MMF (RR 0.64, 95\% CI 0.39-1.06). Of patients on cyclophosphamide, 6/106 developed amenorrhea compared with none on MMF.

\section{Quality of life and economic issues}

Treatment for lupus nephritis has significant cost implications; both in terms of the cost of the medication as well as ancillary factors (eg, staff time and costs, use of available beds). In addition, both the disease and treatment can significantly affect quality of life. There are unfortunately minimal data in this area with only 1 economic model and 1 quality of life study published.

Wilson et al developed a model simulating the costs and outcomes of treating a patient with lupus nephritis with either intravenous cyclophosphamide (dosing schedule based on Ginzler et al 2005; Ong et al 2005) or MMF (mean dose of $2.7 \mathrm{~g}$ daily, based on trial doses between 1-3 g daily) (Wilson et al 2007). Costs were based on the 2005 price-year for the National Health Service (NHS) in the UK. Their results showed that MMF was the less costly induction strategy, costing $£ 1,388$ over the 24 -week period, compared with $£ 2,994$ for cyclophosphamide. In addition, treatment with MMF resulted in superior quality of life with 0.26 QALYs compared with 0.22 for cyclophosphamide. Tse et al published the only available study of a quality of life comparison between MMF and cyclophosphamide treatment for lupus nephritis (Tse et al 2006). This was in 12 patients and overall, MMF treatment was associated with higher domain scores in both the instruments used (SF36 and WHOQOL).

Both these studies have limitations. The economic model uses inferred rather than directly measured quality of life data to derive QALYs, aggregates data from randomized controlled trials of limited quality to generate models that might not necessarily be representative of long-term costs or directly obtained health resource utilization; and uses predominantly US treatment data with UK economic costing data. The quality of life study was retrospectively done in a small group of patients where there are insufficient data to draw any definitive conclusions about the efficacy of MMF vs cyclophosphamide.

It has also been suggested that a reduced side effect rate and improved quality of life with MMF might improve compliance (Tse et al 2006), but there are no direct data comparing this between the two agents. It is possible that compliance could be more challenging with long-term daily oral medication, as opposed to intermittent intravenous cyclophosphamide in a hospital/clinic setting. Particularly in adolescent patients, it has been noted that some physicians might be reluctant to entrust them with responsibility for their induction treatment (Paredes 2007).

In summary, good quality data are still much needed with respect to cost effectiveness, quality of life and compliance issues.

\section{Unresolved questions Use in severe renal impairment}

There are very limited data on the use of MMF in lupus nephritis where renal impairment is severe. All major trials to date excluded patients with severe renal impairment and, consequently, it will be of interest to see the final results from the Aspreva Lupus Management Study which excluded only patients on dialysis but not others with severe renal impairment.

\section{Long-term data}

Compared with the 20-30 years of experience with intravenous cyclophosphamide, there are limited available data on the longer-term outcomes and safety profile of MMF. Although short-term data on MMF are generally favorable, further studies are required to delineate the longer-term relapse rates and risk of renal disease progression while on MMF immunosuppression especially as end-stage renal failure can occur up to 5-10 years after diagnosis.

\section{MMF dosing}

At present, the optimal dose of MMF and the duration of induction therapy are unknown. The 6-month induction phase used in most studies is derived from the experience with intravenous cyclophosphamide. However, few data are available to indicate if 6 months of therapy is ideal or whether a more prolonged period would be appropriate depending on response to therapy.

In addition, the dose of MMF in trials was not modified depending on levels of free MMF in plasma. There are multiple factors associated with lupus nephritis (eg, hypoalbuminemia, renal impairment, concomitant medication, hemoglobin) which can affect the pharmacokinetics. In the transplantation setting, there are data to suggest that MMF levels are associated with efficacy and safety (van Gelder et al 1999; Weber et al 2002; Le Meur et al 2007). Studies in the 
transplant setting have also reported that increased plasma levels of MMF correlated with hematological, infectious and gastrointestinal side effects, although there were differences depending on which pharmacokinetic parameter was used (reviewed in van Gelder and Shaw 2005).

There are limited data about measurement of MMF plasma levels in lupus nephritis at present, although it would seem reasonable to extrapolate the data from the transplant setting which suggests that therapeutic drug monitoring of MMF is likely to be of benefit. Further studies, though, are currently underway in the transplant setting to determine more exactly the advantages of MMF monitoring.

\section{Conclusion}

Lupus nephritis represents a significant complication of SLE with substantial morbidity and mortality. Although treatment has improved, the current standard of therapy with cyclophosphamide is associated with significant morbidity, including an increased risk of serious infections and amenorrhea. MMF has emerged as a potential alternative treatment, both in induction and maintenance therapy for lupus nephritis. However, the randomized trials and cohort studies published to date have all reported on small numbers of patients, with the largest study including 140 patients. Only 1 randomized controlled study showed that MMF was statistically superior to cyclophosphamide for induction. The remainder of the controlled trials did show slightly better results with MMF compared with cyclophosphamide but due to the small numbers of patients recruited, none were sufficiently powered to demonstrate a significant difference, lending credence to the suggestion that MMF might be superior to cyclophosphamide. Unfortunately, the induction phase of the ALMS study which has recently been reported has not contributed further knowledge to the discussion as to whether MMF is or is not superior to cyclophosphamide, although the data from the maintenance phase are now awaited.

One point of note is that these trials included a significant number of non-Caucasian patients, raising the possibility that their results might have greater general application. In view of these small studies, several meta-analyses have been carried out. Again, these showed varying results depending on which trials were included in the meta-analysis, although they all suggest that MMF is at least as effective if not more so than cyclophosphamide. For adverse events, again there are difficulties in making definitive conclusions due to the small numbers, although it would seem that treatment with MMF was less likely to result in death, significant infection, amenorrhea, or leucopenia than cyclophosphamide, although these differences did not always reach statistical significance. Diarrhea appeared to be more common with MMF.

A limited number of studies have investigated the quality of life and economic benefits associated with MMF compared with cyclophosphamide. These have been favorable, although of limited quality with small numbers of patients and limited data available for modeling. Nonetheless, MMF would be expected to result in better quality of life for patients in view of its possible reduced adverse event profile.

It would seem that so far, MMF represents a potential development for the treatment of lupus nephritis, although several questions remained unanswered. No comparison has been made with MMF and the low-dose intravenous cyclophosphamide regimen (Houssiau et al 2002), which has been shown to be as efficacious as the high-dose regimen with potentially fewer side effects. In addition, the studies on MMF published to date have been relatively small with limited follow-up duration, making it difficult to assess the effect of MMF on the clinically important outcomes of death and end-stage renal disease. Longer-term data and experience with MMF are definitely still needed to fully assess what role it will play in the management of lupus nephritis.

\section{Disclosures}

Neither author has any conflicts of interest to disclose.

\section{References}

Adams A, MacDermott EJ, Lehman TJ. 2006. Pharmacotherapy of Lupus Nephritis in Children: A Recommended Treatment Approach. Drugs, 66:1191-207.

Allison AC, Almquist SJ, Muller CD, et al. 1991. In vitro immunosuppressive effects of mycophenolic acid and an ester pro-drug, RS-61443. Transplant Proc, 23:10-4.

Allison AC, Eugui EM. 2000. Mycophenolate mofetil and its mechanisms of action. Immunopharmacology, 47:85-118.

Austin HA, Illei GG. 2005. Membranous lupus nephritis. Lupus, 14:65-71.

Austin III HA, Klippel JH, Balow JE, et al. 1986. Therapy of lupus nephritis. Controlled trial of prednisone and cytotoxic drugs. N Engl J Med, 314: 614-9.

Bastian HM, Roseman JM, McGwin G Jr et al. for the LUMINA Study Group. 2002. Systemic lupus erythematosus in three ethnic groups. XII. Risk factors for lupus nephritis after diagnosis. Lupus, 11:152-60.

Blaheta RA, Leckel K, Wittig B, et al. 1999. Mycophenolate mofetil impairs transendothelial migration of allogeneic CD4 and CD8 T cells. Transplant Proc, 31:1250-2.

Boumpas DT, Austin III HA, Vaughn EM, et al. 1992. Controlled trial of pulse methylprednisolone versus two regimens of pulse cyclophosphamide in severe lupus nephritis. Lancet, 340:741-5.

Budde K, Knoll G, Curtis J. 2006. Long-term safety and efficacy after conversion of maintenance renal transplant recipients from mycophenolate mofetil (MMF) to enteric-coated mycophenolate sodium (EC-MPA, myfortic). Clin Nephrol, 66:103-11.

Bullingham RE, Nicholls AJ, Kamm BR. 1998. Clinical pharmacokinetics of mycophenolate mofetil. Clin Pharmacokinet, 34: 429-55.

Buratti S, Szer S, Spencer CH, et al. 2001. Mycophenolate mofetil treatment of severe renal disease in pediatric onset systemic lupus erythematosus. J Rheumatol, 28:2103-8. 
Cameron JS. 1999. Lupus nephritis. J Am Soc Nephrol, 10:413-24.

Carr SF, Papp E, Wu JC, et al. 1993. Characterization of human type I and type II IMP dehydrogenases. J Biol Chem, 268:27286-90.

Chan L, Mulgaonkar S, Walker R, et al. 2006. Patient-reported gastrointestinal symptom burden and health-related quality of life following conversion from mycophenolate mofetil to enteric coated mycophenolate sodium. Transplantation, 81:1290-7.

Chan TM, Li FK, Tang CS, et al. 2000. Efficacy of mycophenolate mofetil in patients with diffuse proliferative lupus nephritis. Hong KongGuangzhou Nephrology Study Group. N Engl J Med, 343:1156-62.

Chan TM, Tse KC, Tang CS, et al. 2005. Long-term study of mycophenolate mofetil as continuous induction and maintenance treatment for diffuse proliferative lupus nephritis. J Am Soc Nephrol, 16:1076-84.

Colic M, Stojic-Vukanic Z, Pavlovic B, et al. 2003. Mycophenolate mofetil inhibits differentiation, maturation and allostimulatory function of human monocyte-derived dendritic cells. Clin Exp Immunol, 134:63-9.

Contreras G, Pardo V, Leclercq B, et al. 2004. Sequential therapies for proliferative lupus nephritis. N Engl J Med, 350:971-80.

Contreras G, Tozman E, Nahar N, et al. 2005. Maintenance therapies for proliferative lupus nephritis: mycophenolate mofetil, azathioprine and intravenous cyclophosphamide. Lupus, 14(Suppl 1):S33-8.

Corna D, Morigi M, Facchinetti D, et al. 1997. Mycophenolate mofetil limits renal damage and prolongs life in murine lupus autoimmune disease. Kidney Int, 51:1583-9.

Danchenko N, Satia JA, Anthony MS. 2006. Epidemiology of systemic lupus erythematosus: a comparison of worldwide disease burden. Lupus, 15:308-18.

EMEA (European Medicines Agency). 2008. Cellcept, European public assessment report 2008 [online]. Accessed 1 Feburary 2008. URL: http:/www.emea.europa.eu/humandocs/Humans/EPAR/cellcept/ cellcept.htm

Flanc RS, Roberts MA, Strippoli GF, et al. 2004. Treatment of diffuse proliferative lupus nephritis: a metaanalysis of randomized controlled trials. Am J Kidney Dis, 43:197-208.

Flores-Suárez LF, Villa AR. 2004. Preliminary results of an open label randomised clinical trial comparing mycophenylate mofetil (MMF) vs intravenous cyclophosphamide as induction therapy for severe lupus nephritis. Arthritis Rheum, 50(Suppl):S414, 1029

Fu YF, Liu GL. 2001. Mycophenolate mofetil therapy for children with lupus nephritis refractory to both intravenous cyclophosphamide and cyclosporine. Clin Nephrol, 55:318-21.

Furness PN, Taub N. 2006. Interobserver reproducibility and application of the ISN/RPS classification of lupus nephritis - A UK-wide study. Am J Surg Pathol, 30:1030-5.

Ginzler EM, Appel GB, Dooley MA, et al. 2007. Mycophenolate Mofetil and Intravenous Cyclophosphamide in the Aspreva Lupus Management Study (ALMS): Efficacy by Racial Group. Arthritis Rheum, 54(Suppl Abstract):L13.

Ginzler EM, Dooley MA, Aranow C, et al. 2005. Mycophenolate mofetil or intravenous cyclophosphamide for lupus nephritis. $N$ Engl $J$ Med, 353:2219-28.

Goldblum R. 1993. Therapy of rheumatoid arthritis with mycophenolate mofetil. Clin Exp Rheumatol, 11(Suppl 8):S117-S19.

Gourishankar S, McDermid JC, Jhangri GS, et al. 2004. Herpes zoster infection following solid organ transplantation: incidence, risk factors and outcomes in the current immunosuppressive era. Am J Transplant, $4: 108-15$.

Gourley MF, Austin III HA, Scott D, et al. 1996. Methylprednisolone and cyclophosphamide, alone or in combination, in patients with lupus nephritis. A randomized, controlled trial. Ann Intern Med, 125:549-57.

Houssiau FA, Vasconcelos C, D'Cruz D, et al. 2002. Immunosuppressive therapy in lupus nephritis. Arthritis Rheum, 46:2121-31.

Houssiau FA, Vasconcelos C, D'Cruz D, et al. 2004. Early response to immunosuppressive therapy predicts good renal outcome in lupus nephritis: lessons from long-term followup of patients in the Euro-Lupus Nephritis Trial. Arthritis Rheum, 50:3934-40.
Jonsson CA, Svensson L, Carlsten H. 1999. Beneficial effect of the inosine monophosphate dehydrogenase inhibitor mycophenolate mofetil on survival and severity of glomerulonephritis in systemic lupus erythematosus (SLE)-prone MRLlpr/lpr mice. Clin Exp Immunol, 116:534-41.

Kapitsinou PP, Boletis JN, Skopouli FN, et al. 2004. Lupus nephritis: treatment with mycophenolate mofetil. Rheumatology, (Oxford) 43:377-80.

Karim MY, Pisoni CN, Ferro L, et al. 2005. Reduction of proteinuria with mycophenolate mofetil in predominantly membranous lupus nephropathy. Rheumatology, (Oxford) 44:1317-21.

Kasitanon N, Petri M, Haas M, et al. 2008. Mycophenolate mofetil as the primary treatment of membranous lupus nephritis with and without concurrent proliferative disease: a retrospective study of 29 cases. Lupus, 17:40-5.

Kitiyakara C, Ophascharoensuk V, Changsirikulchai S, et al. 2008. Treatment of lupus nephritis and primary glomerulonephritis with entericcoated mycophenolate sodium. Clin Nephrol, 69:90-101.

Lagaraine C, Lebranchu Y. 2003. Effects of immunosuppressive drugs on dendritic cells and tolerance induction. Transplantation, 75:37S-42S.

Lau KL, Jones DP, Hastings MC, et al. 2006. Short-term outcomes of severe lupus nephritis in a cohort of predominantly African-American children. Pediatr Nephrol, 21:655-62.

Le Meur Y, Büchler M, Thierry A, et al. 2007. Individualized mycophenolate mofetil dosing based on drug exposure significantly improves patient outcomes after renal transplantation. Am J Transplant, 7:2496-503.

Lui SL, Tsang R, Wong D et al. 2002. Effect of mycophenolate mofetil on severity of nephritis and nitric oxide production in lupus-prone MRL/lpr mice. Lupus, 11:411-8.

McMurray RW, Elbourne KB, Lagoo A, et al. 1998. Mycophenolate mofetil suppresses autoimmunity and mortality in the female NZB $\times$ NZW F1 mouse model of systemic lupus erythematosus. $J$ Rheumatol, 25:2364-70.

Moore RA, Derry S. 2006. Systematic review and meta-analysis of randomised trials and cohort studies of mycophenolate mofetil in lupus nephritis. Art Res Ther, 8:R182.

Morath C, Schwenger V, Beimler J, et al. 2006. Antifibrotic actions of mycophenolic acid. Clin Transplant, 20 (Suppl 17):25-9.

Natsumeda Y, Ohno S, Kawasaki H, et al. 1990. Two distinct cDNAs for human IMP dehydrogenase. J Biol Chem, 265: 5292-5.

Nowak I, Shaw LM. 1995. Mycophenolic acid binding to human serum albumin: characterization and relation to pharmacodynamics. Clin Chem, 41:1011-7.

Ong LM, Hooi LS, Lim TO, et al. 2005. Randomized controlled trial of pulse intravenous cyclophosphamide versus mycophenolate mofetil in the induction therapy of proliferative lupus nephritis. Nephrology, (Carlton) 10:504-10.

Paredes A. 2007. Can mycophenolate mofetil substitute cyclophosphamide treatment of pediatric lupus nephritis? Pediatr Nephrol, 22:1077-82.

Patel M, Clarke AM, Bruce IN, et al. 2006. The prevalence and incidence of biopsy-proven lupus nephritis in the UK: Evidence of an ethnic gradient. Arthritis Rheum, 54:2963-9.

Pecoraro C, Malgieri G, D'Armiento M, et al. 2005. Treatment of childhood lupus nephritis with mycophenolate mofetil: clinical and histopathological study. J Am Soc Nephrol, 16:557A.

Perez-Aytes A, Ledo A, Boso V, et al. 2008. In utero exposure to mycophenolate mofetil: a characteristic phenotype? Am J Med Genet, 146:1-7.

Ramos MA, Pinera C, Setien MA, et al. 2003. Modulation of autoantibody production by mycophenolate mofetil: effects on $(\mathrm{NZB} \times \mathrm{NZW}) \mathrm{F} 1$ mice. Nephrol Dial Transplant, 18:878-83.

Roos N, Poulalhon N, Farge D, et al. 2007. In vitro evidence for a direct antifibrotic role of the immunosuppressive drug mycophenolate mofetil. J Pharmacol Exp Ther, 321:583-9.

Salvadori M, Holzer H, de Mattos A, et al. 2004. Enteric-coated mycophenolate sodium is therapeutically equivalent to mycophenolate mofetil in de novo renal transplant patients. Am J Transplant, 4:231-6. 
Senda M, DeLustro B, Eugui E, et al. 1995. Y. Mycophenolic acid, an inhibitor of IMP dehydrogenase that is also an immunosuppressive agent, suppresses the cytokine-induced nitric oxide production in mouse and rat vascular endothelial cells. Transplantation, 60:1143-8.

Sinclair A, Appel G, Dooley MA, et al. 2007. Mycophenolate mofetil as induction and maintenance therapy for lupus nephritis: rationale and protocol for the randomized, controlled Aspreva Lupus Management Study (ALMS). Lupus, 16:972-80.

Song AT, Abdala E, Bonazzi PR, et al. 2006. Does mycophenolate mofetil increase the risk of cytomegalovirus infection in solid organ transplant recipients?A mini-review. Braz J Infect Dis, 10:132-8.

Spetie DN, Tang Y, Rovin BH, et al. 2004. Mycophenolate therapy of SLE membranous nephropathy. Kidney Int, 66:2411-5.

Trager J, Ward MM. 2001. Mortality and causes of death in systemic lupus erythematosus. Curr Opin Rheumatol, 13:345-51.

Tse KC, Tang CSO, Lio WI, et al. 2006. Quality of life comparison between corticosteroid-and-mycofenolate mofetil and corticosteroid-and-oral cyclophosphamide in the treatment of severe lupus nephritis. Lupus, 15:371-9.

Van Bruggen MC, Walgreen B, Rijke TP, et al. 1998. Attenuation of murine lupus nephritis by mycophenolate mofetil. J Am Soc Nephrol, 9:1407-15.

van Gelder T, Hilbrands LB, Vanrenterghem Y, et al. 1999. A randomized double-blind, multicenter plasma concentration controlled study of the safety and efficacy of oral mycophenolate mofetil for the prevention of acute rejection after kidney transplantation. Transplantation, 68:261-6.

van Gelder T, Shaw LM. 2005. The rationale for and limitations of therapeutic drug monitoring for mycophenolate mofetil in transplantation. Transplantation, 80(2 Suppl):S244-53.

van Hest RM, Mathot RA, Pescovitz MD, et al. 2006. Explaining variability in mycophenolic acid exposure to optimize mycophenolate mofetil dosing: a population pharmacokinetic meta-analysis of mycophenolic acid in renal transplant recipients. J Am Soc Nephrol, 17:871-80.
Walsh M, James M, Jayne D, et al. 2007. Mycophenolate mofetil for induction therapy of lupus nephritis: a systematic review and metaanalysis. Clin J Am Soc Nephrol, 2:968-75.

Wang K, Zhang H, Li Y, et al. 2004. Safety of mycophenolate mofetil versus azathioprine in renal transplantation: a systematic review. Transplant Proc, 36:2068-70.

Weber LT, Shipkova M, Armstrong VW, et al. 2002. The pharmacokinetic-pharmacodynamic relationship for total and free mycophenolic acid in pediatric renal transplant recipients: A report of the German Study Group on Mycophenolate Mofetil Therapy. J Am Soc Nephrol, 13:759-68.

Weening JJ, D'Agati VD, Schwartz MM, et al. 2004. The classification of glomerulonephritis in systemic lupus erythematosus revisited. $J \mathrm{Am}$ Soc Nephrol, 15:241-50.

Wilson ECF, Jayne DRW, Dellow E, et al. 2007. The cost-effectiveness of mycophenolate mofetil as firstline therapy in active lupus nephritis. Rheumatology, 46:1096-101.

Ye Z, Tan YH, Hong XP, et al. 2001. MMF vs CTX in the treatment of severe SLE patients. Lupus, 10(Suppl 1):S99, 274.

Yokoyama H, Wada T, Hara A, et al. 2004. The outcome and a new ISN/ RPS 2003 classification of lupus nephritis in Japanese. Kidney Int, 66:2382-8.

Yu CC, Yang CW, Wu MS, et al. 2001. Mycophenolate mofetil reduces renal cortical inducible nitric oxide synthase mRNA expression and diminishes glomerulosclerosis in MRL/lpr mice. J Lab Clin Med, 138:69-77.

Zhu B, Chen N, Lin Y, et al. 2007. Mycophenolate mofetil in induction therapy of severe lupus nephritis: a meta-analysis of randomized controlled trials. Nephrol Dial Transplant, 22:1933-42.

Zoja C, Benigni A, Noris M, et al. 2001. Mycophenolate mofetil combined with a cyclooxygenase- 2 inhibitor ameliorates murine lupus nephritis. Kidney Int, 60:653-63. 